\title{
Link adaptation for energy-efficient uplink coordinated multi-point receptions
}

\author{
Young-Han Nam ${ }^{1 *}$, Lingjia Liu ${ }^{1,2}$, Guowang Miao ${ }^{1}$ and Charlie Jianzhong Zhang ${ }^{1}$
}

\begin{abstract}
We investigate link adaptation methods for energy-efficient uplink coordinated multi-point receptions. A system model for practical cellular networks is introduced, in which only a subset of base stations participates in cooperative link adaptation and cooperative decoding for uplink transmissions. To cope with channel-stateinformation (CSI) mismatch incurred from the system model, link adaptation controllers implementing rate back-off from the maximum achievable rate calculated with the mismatched CSI is introduced. From analytical and simulation results, it is concluded that under a certain condition, the rate back-off does not help to improve energy efficiency, where, for example, the condition holds when the CSI errors are modeled as additive Gaussian random variables. Furthermore, energy efficiency of multi-user spatial-division-multiple-access uplink transmissions is studied in isolated cooperative cellular networks. In this scenario, an analytical expression for the optimal link adaptation achieving maximum energy efficiency is obtained.
\end{abstract}

Keywords: coordinated multipoint receptions, CoMP, energy efficiency

\section{Introduction}

Spectral efficiency and energy efficiency are important metrics for wireless communication systems. While contemporary wireless telecommunications standards, e.g., LTE-Advanced mainly focuses on enhancing spectral efficiency (e.g., [1]), there is growing interest in improving energy efficiency, partly because the development of battery technology has not kept in pace with the demand of mobile communications [2,3]. Energy-efficient communications also tend to reduce electromagnetic interference and lessen environmental impacts, for example, heat dissipation and electronic pollution. Therefore, recent research starts to focus on energy-efficient communication techniques [4-9].

Coordinated multi-point (CoMP) transmissions/reception is one example scheme studied in LTE-Advanced [1] primary targeting on increasing cell-edge user equipment's (UE's) spectral efficiency. In CoMP transmissions, multiple base stations coordinate their transmissions so that served UEs receive data streams with higher downlink (DL) spectral efficiency. In CoMP

\footnotetext{
* Correspondence: ynam@sta.samsung.com

'Dallas Telecomm. R\&D Center, Samsung Telecomm. America, Dallas, Texas, 75082, USA
}

Full list of author information is available at the end of the article receptions, multiple base stations coordinate reception/ decoding of packets from UEs to achieve higher uplink (UL) spectral efficiency.

While there have been lots of researches on throughput improvement of CoMP (for UL CoMP refer to [10] and references therein; for DL CoMP refer to $[11,12]$ and references therein), to the authors' best knowledge, little efforts have been put so far on energy-efficient CoMP communications. In this article, we investigate energy-efficient link adaptation for UL CoMP communications in cellular networks. For this purpose, we define an energy-efficiency metric, in a unit of nats/Joule, ${ }^{1}$ by extending the energy-efficiency metric introduced in [9]. The energy-efficiency metric accounts for both transmit power and circuit power. The transmit power models all the power used for reliable data transmission. On the other hand, the circuit power represents the average power consumption of device electronics, e.g. filters, mixers, and digital-to-analog converters, and this portion of power consumption excludes that of the power amplifier and is independent of the transmission state. The newly introduced metric can measure energy efficiency of both the single-cell operations and the CoMP operations, despite fundamental differences of available channel state information (CSI). 
In the first part of the article, we investigate the energy efficiency of a CoMP reception scheme of a single UE's uplink transmission in a cellular network, where only a subset of base stations participate in the cooperative decoding, and the other UEs' signals intended to the other base stations in the network may interfere with the single UE's uplink signals. In this scenario, the CSI experienced during the actual transmission may be different from the CSI used for link adaptation, owing to un-coordinated interference from the other UEs.

To cope with the CSI mismatch, we consider a link adaptation controller implementing a rate back-off from the maximum achievable rate calculated with the mismatched CSI. Then, we analyze the energy efficiency of the link adaptation controller and we show that if a certain condition is satisfied, then the link adaptation controller may still rely on the mismatched CSI in order to achieve the maximum energy efficiency.

In the second part of the article, we analyze the optimal energy efficiency of a CoMP reception scheme of multiple UEs' uplink transmission in a cellular network, where all the base stations participate in the cooperative decoding. In this interference-free scenario, we assume that perfect CSI is available at the link adaptation controller and analyzes conditions for achieving the optimal energy efficiency. In particular, we provide analytical expressions for the optimal power allocation for a twoUE two-base-station system.

The notations used in this article are summarized as in the following. Italic characters, e.g., $K, h$, are used for representing scalar variables. Boldface lowercase Roman characters, e.g., h, are used for representing vectors, and boldface uppercase Roman characters, e.g., H, are used for representing matrices. Boldface Italic lowercase characters, e.g., $\boldsymbol{h}$, are used for representing either random variables or random vectors, while boldface Italic uppercase characters, e.g., $\boldsymbol{H}$ are used for representing random matrices. $\mathbf{A}^{\mathbf{H}}$ denotes the Hermitian transpose of matrix A, and $h^{*}$ denotes complex conjugate of a complex scalar $h .|h|$ denotes the absolute value of a complex scalar $h$, and $\|\mathbf{h}\|$ denotes the L2 norm of a complex vector $\mathbf{h}$. $\mathbb{C}$ denotes the set of complex numbers.

\section{System model}

We consider an uplink transmission of $K$ single-antenna UEs in a cellular network composed of $\bar{M}$ base stations. $M(\leq \bar{M})$ clustered base stations in the cellular network cooperate to decode the UEs' uplink signals. For the cooperation, each of the $M$ base stations are connected to a central controller with fiber, where the fiber transmission is assumed to be information lossless. The central controller has a link adaptation controller and a receiver. The link adaptation controller determines transmission rate and transmission power for the UEs based on the available CSI, and commands the UEs to transmit messages with the determined rate and power. On the other hand, the receiver decodes the transmitted messages from received signals. As the $M$ base stations receives interfering signals from the other UEs transmitting to the other $(\bar{M}-M)$ non-cooperating base stations in the cellular network as well as signal components from the $K$ UEs, the channel state, especially the interference state, used for demodulation at the receiver is not necessarily the same as the state reported to the link adaptation controller because of time-varying interfering signals from other non-cooperating users. This discrepancy of CSI is sometimes called flashlight effect in the wireless telecommunications industry [1]. Figure 1 illustrates the system model for $K=1, M=2$ and $\bar{M}=3$, where UE 0 transmits signals to $K=2$ cooperating base stations, while UE 1's signals interferes with UE O's signals at the receiver at the central controller.

Under the system model considered in this section, we consider both single-user and multi-user transmission scenarios with uplink CoMP receptions. In Section 3, we consider energy-efficient uplink CoMP receptions of a single user in a partially cooperating cellular wireless network, in which only a subset of base stations performs cooperation. For single-user transmissions, the further refined system model in Section 3 is general enough to reflect some important aspects of the real-life cellular networks, and at the same time, it is possible to obtain some analytical results. However, for multi-user multi-cell link adaptations, the system model of Section 3 is difficult to analyze. To obtain some insights of multi-user transmission scenarios aided by CoMP

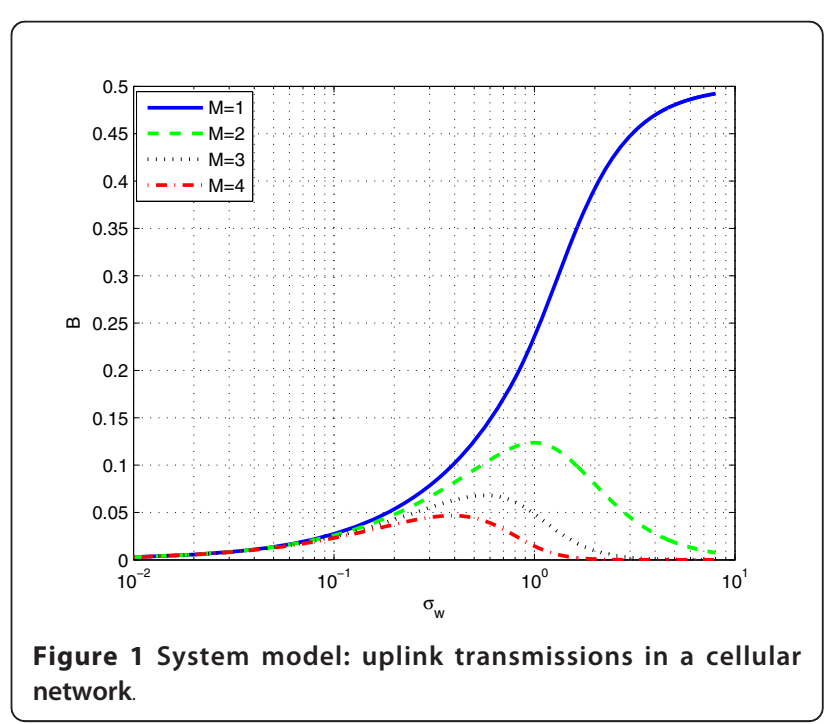


reception, we make further simplifying assumption of fully cooperating cellular networks in Section 4.

\section{Energy-efficient uplink transmission schemes with $K=1$ and $M \ll \bar{M}$}

3.1 Definition of energy efficiency with imperfect CSI

Traditionally, energy efficiency is defined for a single link $(M=1)$, under the assumption that both the link adaptation controller and the receiver are aware of the channel state, and the channel state stays the same during the link adaptation and the reception. Under this assumption, link adaptation controller can always adapt transmission power and rate so that the packet is successfully decoded with probability one according to the Shannon's channel coding theorem [13]. Then, given a channel coefficient $h \in \mathbb{C}$, the energy efficiency for the single link, where a UE always successfully transmits a packet with transmission rate $R$ by spending total power $P=P_{T}+P_{C}$ to a receiver, is defined (e.g., [9]) as

$$
U_{h}\left(P_{T}\right) \triangleq \frac{R\left(P_{T}, h\right)}{P_{T}+P_{C}} \text { nats per Joule }
$$

Assuming capacity achieving channel codes and sufficiently large block length, we have $R\left(P_{T}, h\right)=\ln (1+|h|$ ${ }^{2} P_{T}$ ) nats. The maximal energy efficiency $U_{h}^{*}$ for a given channel coefficient $h$ is achieved with the optimal rate and power allocation $P_{T}^{*}(h)$ :

$$
U_{h}^{*}=\frac{R\left(P_{T}^{*}(h), h\right)}{P_{T}^{*}(h)+P_{C}} \text { and } P_{T}^{*}(h)=\underset{P_{T}}{\arg \max } \frac{R\left(P_{T}, h\right)}{P_{T}+P_{C}} .
$$

Now, once a distribution of the random variable $\boldsymbol{h}$ characterizing $h$ is given, the optimal energy efficiency of the network $U^{\star}$ can be defined as $U^{*} \triangleq \mathbb{E}\left(U_{h}^{*}\right)$.

However, as the channel states used for the link adaptation is not necessarily the same as those for the receiver, we consider a general framework for taking potential imperfectness of CSI at the link adaptation controller and extend the energy efficiency definition accordingly. For the uplink transmission considered in this section, we make the following assumptions.

- (Assumption 1) The actual channel vector of the network during the transmission of a packet is $\mathbf{h} \in$ $\mathbb{C}^{M \times 1}$, and the $m$ th component of $\mathbf{h}$, denoted by $h_{m}$, is the channel coefficient between BS $m$ and the UE. The received signals at each BS are corrupted by circularly symmetric additive white Gaussian noise (AWGN) of zero mean and unit variance. We note that this assumption models a receiver treating interference signals from the other UEs transmitting to the other base stations as noise and scaling the received signal, so that the interference-plus-noise power is one.

- (Assumption 2) The receiver is aware of the actual channel vector $\mathbf{h}$.

- (Assumption 3) The link adaptation controller determines the transmission rate $R$ and the transmission power $P_{T}$ based on CSI $\tilde{\mathbf{h}}=\mathbf{h}-\boldsymbol{w}$, where $\boldsymbol{w} \in$ $\mathbb{C}^{M \times 1}$ is a random vector characterizing the CSI error and models the un-coordinated interference from other UEs in the cellular network. The link adaptation controller is aware ${ }^{2}$ of the distribution of w.

- (Assumption 4) To cope with channel outages, the link adaptation controller applies a power back-off strategy to determine the transmission rate. The controller assumes that the transmission power is $\alpha P_{T}$ where $\alpha \in(0,1]$ for the rate calculation, even though the actual transmission power is $P_{T}$. In this case, the transmission rate $R$ is determined as $R=R\left(P_{T}, \mathbf{h}, \alpha\right)=\ln \left(1+\alpha P_{T}\|\tilde{\mathbf{h}}\|^{2}\right)$.

Under these assumptions, we define expected energy efficiency of the network seen at the link adaptation controller $\tilde{U}_{\tilde{\mathbf{h}}}\left(P_{T}, R\right)$.

$$
\tilde{U}_{\tilde{\mathbf{h}}}\left(P_{T}, R\right) \triangleq \frac{(1-\operatorname{Pr}(O)) R\left(P_{T}, \tilde{\mathbf{h}}, \alpha\right)}{\left(P_{T}+P_{C}\right)} .
$$

Here, the numerator is the average throughput (nats per channel use) achieved with the transmission rate $R\left(P_{T}, \tilde{\mathbf{h}}, \alpha\right)[14]$, and $\operatorname{Pr}(O)$ is the probability of hypothetical channel outage events seen at the link adaptation controller:

$$
\operatorname{Pr}(O)=\operatorname{Pr}\left(R\left(P_{T}, \tilde{\mathbf{h}}, \alpha\right) \geq \bar{R}\left(P_{T}, \tilde{\mathbf{h}},+\boldsymbol{w}\right)\right),
$$

where $\bar{R}\left(P_{T}, \tilde{\mathbf{h}}+\boldsymbol{w}\right)$ is the maximum achievable rate with the hypothetical actual channel $\tilde{\mathbf{h}}+\boldsymbol{w}$, and hence $\bar{R}\left(P_{T}, \tilde{\mathbf{h}}+\boldsymbol{w}\right)=\ln \left(1+P_{T}\|\tilde{\mathbf{h}}+\boldsymbol{w}\|^{2}\right)$. Then, the optimal transmission power and transmission rate $P_{T}^{*}$ and $R^{*}$ with a given $\tilde{h}$ are determined by,

$$
\left(P_{T}^{*}, R^{*}\right)=\underset{P_{T}, R}{\arg \max } \tilde{U}_{\tilde{\mathbf{h}}}\left(P_{T}, R\right),
$$

and the maximal expected energy efficiency with a given $\tilde{h}$ is given by,

$$
\tilde{U}_{\tilde{\mathbf{h}}}^{*}=\tilde{U}_{\tilde{\mathbf{h}}}\left(P_{T}^{*}, R^{*}\right) .
$$


Once a distribution of the random vector $\tilde{h}$ characterizing $\tilde{\mathbf{h}}=\mathbf{h}+\boldsymbol{w}$ is given as well as the distribution of $\boldsymbol{w}$, the optimal energy efficiency $\tilde{U}^{*}$ of the network can be defined as $\tilde{U}^{*} \triangleq \mathbb{E}\left(\tilde{U}_{\tilde{\mathbf{h}}}^{*}\right)$.

\subsection{With perfect CSI at the link adaptation controller}

In this subsection, we consider the uplink transmission with perfect CSI at the link adaptation controller. To model this case, in addition to the four assumptions in Section 3.1, we further assume that the CSI error vector $\boldsymbol{w}$ is deterministically $\mathbf{0}$ so that $\tilde{\mathbf{h}}=\mathbf{h}$, and that the link adaptation controller chooses $\alpha=1$, regardless of the CSI $\mathbf{h}$. In this case, the maximization problem of (6) reduces to (2) and has been analyzed in [9], as in the following theorem.

Theorem 1. The optimal power allocation $P_{T}^{*}$ and the optimal transmission rate $R^{*}$ for the uplink transmission with perfect CSI at the link adaptation controller with a given $\mathbf{h}$ is obtained by the following relation:

$$
P_{T}^{*}=\left(\frac{1}{U_{\mathbf{h}}^{*}}-\frac{1}{\|\mathbf{h}\|^{2}}\right)^{+} \text {and } R^{*}=\ln \left(1+P_{T}^{*}\|\mathbf{h}\|^{2}\right),
$$

where

$$
U_{\mathbf{h}}^{*}=U_{\mathbf{h}}\left(P_{T}^{*}\right)=\frac{\ln \left(1+P_{T}^{*}\|\mathbf{h}\|^{2}\right)}{P_{T}^{*}+P_{C}} .
$$

We note that efficient algorithms are available to solve the power maximization (7), e.g., from [9].

\subsection{With imperfect CSI at the link adaptation controller}

The expected energy efficiency at the link adaptation controller $\tilde{U}_{\tilde{\mathbf{h}}}\left(P_{T}\right)$ of the uplink transmission modeled in Section 3.1 is further expanded as,

$$
\begin{aligned}
\tilde{U}_{\tilde{\mathbf{h}}}\left(P_{T}, \alpha\right) & =\frac{R\left(P_{T}, \tilde{\mathbf{h}}, \alpha\right)}{P_{T}+P_{C}} \cdot(1-\operatorname{Pr}(O)) \\
& =\frac{\ln \left(1+\alpha P_{T}\|\tilde{\mathbf{h}}\|^{2}\right)}{P_{T}+P_{C}} \cdot \operatorname{Pr}\left(\ln \left(1+\left.\alpha P_{T}\|\tilde{h}\|\right|^{2}\right) \leq \ln \left(1+P_{T}\|\tilde{\mathbf{h}}+w\|^{2}\right)\right) \\
& =\frac{\ln \left(1+\alpha P_{T}\|\tilde{\mathbf{h}}\|^{2}\right)}{P_{T}+P_{C}} \cdot \operatorname{Pr}(\|\tilde{\mathbf{h}}+w\| \geq \sqrt{\alpha}\|\tilde{\mathbf{h}}\|) .
\end{aligned}
$$

Denoting the probability density function and cumulative distribution function of random variable $\|\tilde{\mathbf{h}}+\boldsymbol{w}\|$ as $f_{\|\tilde{\mathbf{h}}+w\|}(x)$ and $F_{\|\tilde{\mathbf{h}}+w\|}(x)=\int_{0}^{x} f_{\|\tilde{\mathbf{h}}+w\|}(\xi) d \xi$ respectively, the expected energy efficiency is

$$
\tilde{U}_{\tilde{\mathbf{h}}}\left(P_{T}, \alpha\right)=\frac{\ln \left(1+\alpha P_{T}\|\tilde{\mathbf{h}}\|^{2}\right)}{P_{T}+P_{C}} \cdot\left(1+F_{\|\tilde{\mathbf{h}}+w\|}(\sqrt{\alpha}\|\tilde{\mathbf{h}}\|)\right) .
$$

The maximal expected energy efficiency with this link adaptation controller is obtained through a joint maximization over $P_{T}$ and $\alpha$ :

$$
\tilde{U}_{\tilde{\mathrm{h}}}^{*}=\max _{P_{T}, \alpha} \tilde{U}_{\tilde{\mathrm{h}}}\left(P_{T}, \alpha\right)
$$

We obtain the following theorem on this joint maximization.

Theorem 2. Define $P_{T}^{(1)}$ and $R^{(1)}$ as,

$$
P_{T}^{(1)} \triangleq\left(\frac{1}{\tilde{U}_{\tilde{\mathbf{h}}}\left(P_{T}^{(1)}, 1\right)}-\frac{1}{\|\tilde{\mathbf{h}}\|^{2}}\right)^{+} \text {and } R^{(1)} \triangleq \ln \left(1+P_{T}^{(1)}\|\tilde{\mathbf{h}}\|^{2}\right),
$$

and let $A$ be a positive real number satisfying the following relation:

$$
\frac{A\|\tilde{\mathbf{h}}\|^{2}}{\left(1+A\|\tilde{\mathbf{h}}\|^{2} \ln \left(1+A\|\tilde{\mathbf{h}}\|^{2}\right)\right)}=\frac{f_{\|\tilde{\mathbf{h}}+w\|}(\|\tilde{\mathbf{h}}\|)}{1-F_{\|\tilde{\mathbf{h}}+w\|}(\|\tilde{\mathbf{h}}\|)} .
$$

If $P_{T}^{(1)} \leq A$, then the transmission power and the transmission rate achieving the maximum expected energy efficiency are achieved with $\alpha^{*}=1$ :

$$
P_{T}^{*}=P_{T}^{(1)} \text { and } R^{*}=R^{(1)} .
$$

Otherwise, the transmission power and the transmission rate achieving the maximum expected energy efficiency are achieved with $\alpha^{*}<1$, and $P_{T}^{*}$, and $\alpha^{*}$ satisfies the following relation:

$$
\frac{P_{T}^{*}\|\tilde{\mathbf{h}}\|^{2}}{\left(1+\alpha^{*} P_{T}^{*}\|\tilde{\mathbf{h}}\|^{2}\right) \ln \left(1+\alpha^{*} P_{T}^{*}\|\tilde{\mathbf{h}}\|^{2}\right)}=\frac{f_{\|\tilde{\mathbf{h}}+w\|}\left(\sqrt{\alpha^{*}}\|\tilde{\mathbf{h}}\|\right)}{1-F_{\|\tilde{\mathbf{h}} w\|}\left(\sqrt{\alpha^{*}}\|\tilde{\mathbf{h}}\|\right)} .
$$

Proof. See Appendix A

Theorem 2 states that when a condition of $P_{T}^{(1)} \leq A$ holds, the link adaptation controller can maximize the expected energy efficiency by choosing $P_{T}=P_{T}^{(1)}$ and $R=\ln \left(1+P_{T}^{(1)}\|\tilde{\mathbf{h}}\|^{2}\right)$ with treating $\tilde{\boldsymbol{h}}$ as the actual CSI, where $P_{T}^{(1)}$ can be efficiently found by algorithms introduced in [9]. To see when the condition of $P_{T}^{(1)} \leq A$ holds, we need to take a closer look at $A$, which is a parameter determined dependent on the distribution of $w$.

As an example case, we consider a circularly symmetric Gaussian distribution for each component of $\boldsymbol{w}$, with mean 0 and variance $\sigma_{w}^{2}$. Then, $\|\tilde{\mathbf{h}}+\boldsymbol{w}\|^{2}$ is noncentral chi-square distributed, with degrees of freedom $2 M$, mean $\|\tilde{\mathbf{h}}\|^{2}$ and non-centrality parameter $2\|\tilde{\mathbf{h}}\|^{2} / \sigma_{w}^{2}$. To evaluate $A$ in (13), we further assume that $\|\tilde{\mathbf{h}}\|=1$, $P c=1$ and consider various $\sigma_{w}^{2}$ with $M=1,2,3,4$. We 
first notice that $P_{T}^{(1)} \approx 1.72$ with $\|\tilde{\mathbf{h}}\|=1$ regardless of the value of $\sigma_{w}^{2}$ as $P_{T}^{(1)}$ does not depend on the distribution of $w$. In addition, we define $B \triangleq\left(f_{\|\tilde{\mathbf{h}}+\boldsymbol{w}\|}(\|\tilde{\mathbf{h}}\|)\right) /\left(1-F_{\|\tilde{\mathbf{h}}+\boldsymbol{w}\|}(\|\tilde{\mathbf{h}}\|)\right)$ and evaluate $B$ with these parameters as shown in Figure 2. Since the left hand side of (13) is a decreasing function of $A$, the $A$ meeting the condition (13) gets smaller as $B$ increases. As shown in Figure 2, $B$ is bounded above for a given $M$ and $\sigma_{w}>0$, and hence the minimum $A$ satisfying the condition (13) is greater than the supremum of $B$ obtained with the given $M$ and $\sigma_{w}>0$. For example, when $M=1$, the supremum of $B$ is equal to 0.5 , and $A$ satisfying the condition (13) with $B=0.5$ is $A \simeq e^{2}$, which is greater than $P_{T}^{(1)}=1.72$. Since the supremum of $B$ further reduces as $M$ increases, a greater $A$ is needed to satisfy the condition (13) with the supremum of $B$ as $M$ increases. From these observations, we find that for $M \geq 1$, the condition of $A \geq P_{T}^{(1)}=1.72$ holds for all $\sigma_{w}^{2}>0$, and hence the link adaptation controller can simply assign $P_{T}=P_{T}^{(1)}$ and $R=\ln \left(1+P_{T}^{(1)}\|\tilde{\mathbf{h}}\|^{2}\right)$ without explicitly considering the variance of the CSI error $\sigma_{w}^{2}$ for achieving the maximum expected of the network.

When the condition of $P_{T}^{(1)} \leq A$ holds, the maximum expected energy efficiency of the uplink coordinated multi-point reception with imperfect CSI at the link adaptation controller is

$$
\tilde{U}_{\tilde{\mathbf{h}}}^{*}=\frac{\ln \left(1+P_{T}^{(1)}\|\tilde{\mathbf{h}}\|^{2}\right)}{P_{T}^{(1)}+P_{C}} \cdot\left(1-F_{\|\tilde{\mathbf{h}}+w\|}(\sqrt{\alpha}\|\tilde{\mathbf{h}}\|)\right) .
$$

For example, the resultant maximum expected energy efficiency with $\|\tilde{\mathbf{h}}\|=1, P_{C}=1$ and $\mathbf{w}$ whose elements are distributed with circularly symmetric Gaussian with mean 0 and variance $\sigma_{w}^{2}$ is shown in Figure 3. $\tilde{U}_{\tilde{\mathrm{h}}}^{*}$

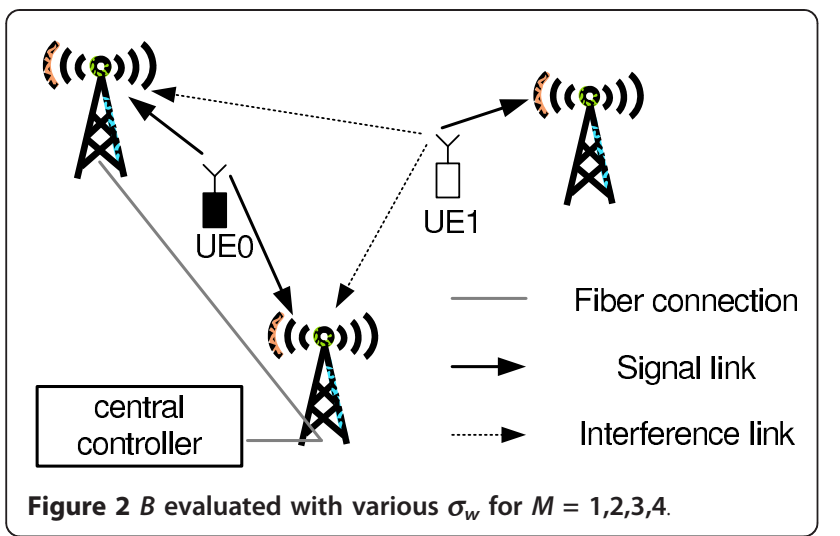

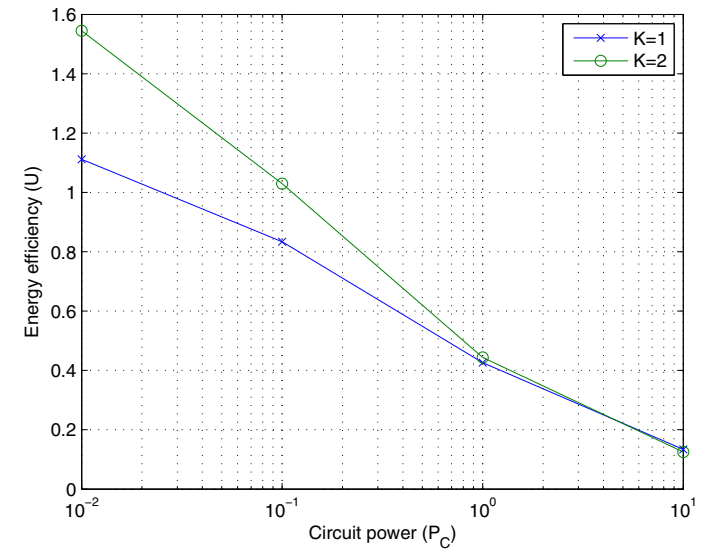

Figure $3 \tilde{U}_{\tilde{\mathrm{h}}}$ evaluated with various $\sigma_{w}$ for $M=1,2,3,4$

decreases as $\sigma_{w}$ increases and it increases as the number of cooperating base stations $M$ increases.

In general, given a distribution of $\tilde{h}$, which is a random vector characterizing $\tilde{\mathbf{h}}$, the maximum expected energy efficiency $\tilde{U}^{*}=\mathbb{E}\left(\tilde{U}_{\tilde{h}}^{*}\right)$ increases as we increase the $M$ number of base stations participating in the cooperative link adaptation and decoding, because of the increased transmission rates and smaller outage probability for a given transmission rate thanks to the diversity reception of the coordinated multi-point reception.

\section{Energy-efficient uplink transmission schemes with $K \geq 1$ and $\bar{M}=M$}

To get some insights of the possible advantages of employing uplink multi-user transmissions in a CoMP setting, we consider a relaxed setting of an isolated cellular network of $M$ cooperating base stations in this section. In the isolated network, the uplink transmissions do not suffer from flash-light effect, and hence both link adaptation controller and the receiver have perfect knowledge of the actual channel states. To model this case, we make the following assumptions:

- (Assumption 1) The actual channel matrix of the network during the transmission of a packet is $\mathbf{H} \in$ $\mathbb{C}^{M \times K}$, and the $(m, k)$ component of $\mathbf{H}$, denoted by $h_{m k}$, is the channel coefficient between BS $m$ and UE $k$, where $m \in\{1, \ldots, M\}$ and $k \in\{1, \ldots, K\}$. The received signals at each BS are corrupted by circularly symmetric additive white Gaussian noise (AWGN) of zero mean and unit variance.

- (Assumption 2) The receiver is aware of the actual channel matrix $\mathbf{H}$.

- (Assumption 3) The link adaptation controller determines the $K$ UE's transmission powers $\left\{P_{T, 1}, \ldots, P_{T}\right.$, $\left.{ }_{K}\right\}$ and rates $\left\{R_{1}, \ldots, R_{K}\right\}$ based on the perfect CSI $\mathbf{H}$, 
such that the maximum sum rate is achieved with the selected powers when either successive interference cancellation (SIC) or joint maximum-likelihood (ML) decoding is used at the central controller.

From the capacity region of the Gaussian multipleaccess channel [13], the maximum sum rate is given by

$$
\sum_{k} R_{k}=\log \operatorname{det}\left(I+\sum_{k} P_{T, k} \mathbf{h}_{k} \mathbf{h}_{k}^{\mathrm{H}}\right)
$$

where $\mathbf{h}_{k}=\left[h_{1 k}, \ldots, h_{\mathrm{M} k}\right]$. In this case, the energy efficiency of the cellular network with channel matrix $\mathbf{H}$ is defined by,

$$
U_{\mathbf{H}}(\mathbf{p}) \triangleq \frac{\log \operatorname{det}\left(I+\sum_{k} P_{T, k} \mathbf{h}_{k} \mathbf{h}_{k}^{\mathrm{H}}\right)}{\sum_{k}\left(P_{T, k}+P_{C, k}\right)}
$$

where $\mathbf{p}=\left[P_{1}, \ldots, P_{K}\right]=\left[P_{T, 1}+P_{C, 1}, \ldots, P_{T, k}+P_{C, k}\right]$. Then the optimal power control to achieve the maximum energy efficiency and the optimal energy efficiency can be found as,

$$
\mathbf{p}^{*}(\mathbf{H})=\underset{\mathbf{p}}{\arg \max } U_{\mathbf{H}}(\mathbf{p}) \quad \text { and } \quad U_{\mathbf{H}}^{*}=U_{\mathbf{H}}\left(P^{*}(\mathbf{H})\right)
$$

When the distribution of a random matrix $\boldsymbol{H}$ characterizing $\mathbf{H}$ is given, we further define the optimal energy efficiency of the network as $U^{*}=E\left(U_{H}^{*}\right)$.

When $K=1$, the optimal link adaptation parameters $P_{T, 1}^{*}$ and $R_{1}^{*}$ can be found as in Theorem 1 . On the other hand, when $K \geq 1$, we can prove the following lemma for the optimization problem (19).

Lemma 3. $U_{\mathbf{H}}(\mathrm{p})$ defined in (18) is strictly quasi-concave in $\mathbf{p}$.

Proof. As log det $\left(I+\sum_{k} P_{T, k} \mathbf{h}_{k} \mathbf{h}_{k}^{\mathrm{H}}\right)$ is concave in $\mathbf{p}$, it can be straightforwardly verified that (18) is strictly quasi-concave in $\mathbf{p}$.

If a strictly quasi-concave function has a local maximum, then the local maximum is global maximum. Relying on this property of $U_{\mathbf{H}}(\mathbf{p})$, we prove the following.

Theorem 4. The optimal power control $\mathbf{p}=\mathbf{p}^{*}$ is found by solving the following system of equations:

$$
\begin{aligned}
& U_{\mathbf{H}}(\mathbf{p})=\mathbf{h}_{1}^{\mathrm{H}}\left(I+\sum_{k} P_{T, k} \mathbf{h}_{k} \mathbf{h}_{k}^{\mathrm{H}}\right)^{-1} \mathbf{h}_{1,} \\
& U_{\mathbf{H}}(\mathbf{p})=\mathbf{h}_{2}^{\mathrm{H}}\left(I+\sum_{k} P_{T, k} \mathbf{h}_{k} \mathbf{h}_{k}^{\mathrm{H}}\right)^{-1} \mathbf{h}_{2}, \\
& U_{\mathbf{H}}(\mathbf{p})=\mathbf{h}_{K}^{\mathrm{H}}\left(I+\sum_{i} P_{T, i} \mathbf{h}_{k} \mathbf{h}_{k}^{\mathrm{H}}\right)^{-1} \mathbf{h}_{K} .
\end{aligned}
$$

Proof Taking the first order partial derivative of $U_{\mathbf{H}}(\mathbf{p})$ on $p_{1}^{T}$, we obtain,

$$
\frac{\partial U(\mathbf{p})}{\partial p_{1}^{T}}=\frac{\operatorname{tr}\left(\left(I+\sum_{k} P_{T, k} \mathbf{h}_{\mathbf{h}} \mathbf{h}_{k}^{\mathrm{H}}\right)^{-1} \mathbf{h}_{\mathbf{1}} \mathbf{h}_{1}^{\mathrm{H}}\right) \sum_{\mathrm{i}}\left(P_{T, k}+P_{C, k}\right)-\log \operatorname{det}\left(I+\sum_{k} P_{T, k} \mathbf{h}_{k} \mathbf{h}_{k}^{\mathrm{H}}\right)}{\left(\sum_{k}\left(P_{T, k}+P_{C, k}\right)\right)^{2}},
$$

Here we notice that $\left(\left(I+\sum_{k} P_{T, k} \mathbf{h}_{k} \mathbf{h}_{k}^{\mathrm{H}}\right)^{-1} \mathbf{h}_{1} \mathbf{h}_{1}^{\mathrm{H}}\right)=\mathbf{h}_{1}^{\mathrm{H}}\left(I+\sum_{i} P_{T, k} \mathbf{h}_{\mathbf{h}} \mathbf{h}_{k}^{\mathrm{H}}\right)^{-1} \mathbf{h}_{1}$. Furthermore, by equating the partial derivative with 0 , we obtain,

$$
\log \operatorname{det}\left(I+\sum_{k} P_{T, k} \mathbf{h}_{\mathbf{h}} \mathbf{H}_{k}^{\mathrm{H}}\right)=\mathbf{h}_{1}^{\mathrm{H}}\left(I+\sum_{k} P_{T, k} \mathbf{h}_{k} \mathbf{h}_{k}^{\mathrm{H}}\right)^{-1} \mathbf{h}_{1} \sum_{i}\left(P_{T, k}+P_{C, k}\right),
$$

or $U_{\mathbf{H}}(\mathbf{p})=\mathbf{h}_{1}^{\mathrm{H}}\left(I+\sum_{k} P_{T, k} \mathbf{h}_{k} \mathbf{h}_{k}^{\mathrm{H}}\right)^{-1} \mathbf{h}_{1}$. Similarly, taking partial derivatives of $U_{\mathbf{H}}(\mathbf{p})$ on $p_{T, 2}, \ldots, p_{T, K}$, we obtain the system of equations giving the optimal power allocation.

Some insights of the power control equations in Theorem 2 can be obtained with analyzing $K=M=2$. With $\mathbf{h}_{1}=\left[h_{11} h_{12}\right]^{\mathrm{T}}$ and $\mathbf{h}_{2}=\left[h_{21} h_{22}\right]^{\mathrm{T}},(20)$ is further simplified as,

$$
\begin{aligned}
U_{\mathbf{H}}(\mathbf{p})=\mathbf{h}_{1}^{\mathrm{H}}\left(I+\sum_{i} P_{T, i} \mathbf{h}_{i} \mathbf{h}_{i}^{\mathrm{H}}\right)^{-1} \mathbf{h}_{1,} \\
=\left[\begin{array}{ll}
\left.h_{11} h_{12}\right]\left[\begin{array}{cc}
1+P_{T, 1}\left|h_{11}\right|^{2}+P_{T, 2}\left|h_{21}\right|^{2} & P_{T, 1} h_{11} h_{12}^{*}+P_{T, 2} h_{21} h_{22}^{*} \\
P_{T, 1} h_{11}^{*} h_{12}+P_{T, 2} h_{21}^{*} h_{22} & 1+P_{T, 1}\left|h_{12}\right|^{2}+P_{T, 2}\left|h_{22}\right|^{2}
\end{array}\right]^{-1}\left[\begin{array}{c}
h_{11}^{*} \\
h_{12}^{*}
\end{array}\right] \\
=\frac{1}{\left(1+P_{T, 1}|| \mathbf{h}_{1}\left\|^{2}+P_{T, 2}|| \mathbf{h}_{2}\right\|^{2}+P_{T, 1} P_{T, 2}|\operatorname{det}(\mathbf{H})|^{2}\right)}\left[h_{11} h_{12}\right.
\end{array}\right] . \\
{\left[\begin{array}{cc}
1+P_{T, 1}\left|h_{21}\right|^{2}+P_{T, 2}\left|h_{22}\right|^{2} & -\left(P_{T, 1} h_{11} h_{12}^{*}+P_{T, 2} h_{21} h_{22}^{*}\right) \\
-\left(P_{T, 1} h_{11}^{*} h_{12}+P_{T, 2} h_{21}^{*} h_{22}\right) & 1+P_{T, 1}\left|h_{11}\right|^{2}+P_{T, 2}\left|h_{21}\right|^{2}
\end{array}\right]\left[\begin{array}{l}
h_{11}^{*} \\
h_{12}^{*}
\end{array}\right] } \\
=\frac{|| \mathbf{h} \|^{2}+P_{T, 2}|\operatorname{det}(\mathbf{H})|^{2}}{\left(1+P_{T, 1}|| \mathbf{h}_{1}||^{2}+P_{T, 2}|| \mathbf{h}_{2} \|\left.\right|^{2}+P_{T, 1} P_{T, 2}|\operatorname{det}(\mathbf{H})|^{2}\right)}
\end{aligned}
$$

Similarly, (21) is further simplified as,

$$
U_{\mathbf{H}}(\mathbf{p})=\frac{\left\|\mathbf{h}_{2}\right\|^{2}+P_{T, 1}|\operatorname{det}(\mathbf{H})|^{2}}{\left(1+P_{T, 1}|| \mathbf{h}_{1}\left\|^{2}+P_{T, 2}|| \mathbf{h}_{2}\right\|^{2}+P_{T, 1} P_{T, 2}|\operatorname{det}(\mathbf{H})|^{2}\right)}
$$

From (28) and (29), we obtain the following relation between $P_{T, 1}$ and $P_{T, 2}$.

$$
P_{T, 2}=\max \left(P_{T, 1}+\frac{\left\|\mathbf{h}_{2}\right\|^{2}-\left\|\mathbf{h}_{1}\right\|^{2}}{|\operatorname{det}(\mathbf{H})|^{2}}, 0\right) .
$$

This relation implies that the link adaptation controller should assign more power to the user with better channel, which gives us a similar intuition as the waterfilling power control for parallel channels [13].

When substituting (30) to (19), the joint optimization (19) over two variables $P_{T, 1}$ and $P_{T, 2}$ is simplified into an easier optimization over a single variable $P_{T, 1}$.

Figure 4 shows the optimal energy efficiency $U^{*}$ over different values of the circuit power $P_{C}$ evaluated for single-user $(K=1)$ and multi-user $(K=2)$ uplink 


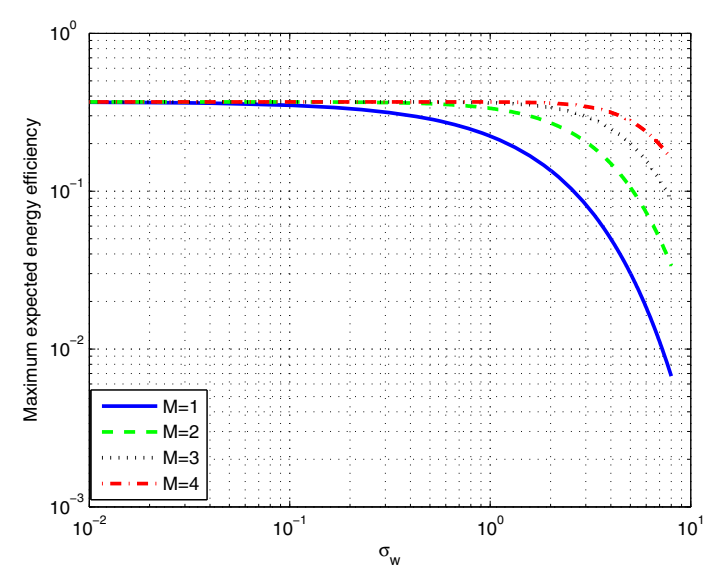

Figure 4 Energy efficiency evaluated with $K=1$ and $K=2$ with various $P_{C}$

transmissions in a isolated cellular network composed of two base stations $(M=2)$. For the evaluation, statistically independent circularly symmetric Gaussian channels are generated with zero mean and unit variance for the links. From the figure, we observe that multi-user transmissions are more energy-efficient than single-user transmissions; thanks to the multiplexing gain of the effective multi-input-multi-output (MIMO) channel. On the other hand, we also observe that the multi-user energy-efficiency gain decreases as the circuit power $P_{c}$ increases. This is partly because when the circuit power $P_{c}$ is large, the denominator of the energy efficiency (18) is dominated by the circuit power.

\section{Conclusions}

In this article, we investigated the energy efficiency of CoMP link adaptations and CoMP reception schemes of uplink transmission in a cellular network. To model typical implementation of CoMP in the cellular network, we first considered a scenario where only a subset of base stations participate in the cooperative link adaptation and cooperative decoding for a single UE, in which case, the other UEs' signals intended to the other base stations in the network interfere with the single UE's uplink signals, and hence the CSI experienced during the actual transmission is different from the CSI used for link adaptation. To cope with the CSI mismatch, we consider a link adaptation controller implementing a rate back-off from the maximum achievable rate calculated with the mismatched CSI. According to the analysis, we found that the maximum energy efficiency of the link adaptation controller is achieved when no rate back-off is employed, when a certain condition is satisfied. We also showed by simulation that the condition holds when the CSI errors are modeled as additive Gaussian random variables. Furthermore, in order to see benefits of multi-user uplink transmissions for energy efficiency, we analyzed the optimal energy efficiency of a link adaptation method and a CoMP reception scheme of multiple UEs' uplink transmissions in a cellular network, where all the base stations participate in the cooperative decoding. In this interference-free or perfect-CSI scenario, we obtained analytical expressions for the optimal power control with arbitrary numbers of UEs and base stations. The optimal power allocation gives similar intuition as water-filling in parallel channels, that is, we need to assign more power to a better-quality channel, to achieve the maximum energy efficiency We also provided a simulation result showing that twouser spatial-division-multiple-access transmissions achieves larger maximum energy efficiency than singleuser transmissions in the same cellular network.

\section{Appendix A}

\section{Proof of Theorem 2}

We first write the maximization problem as in the following:

$$
\tilde{U}_{\tilde{h}}^{*}=\max _{P_{T, \alpha}} \tilde{U}_{\tilde{h}}\left(P_{T}, \alpha\right)=\max _{P_{T}}\left(\max _{\alpha} \tilde{U}_{\tilde{h}}\left(P_{T}, \alpha\right)\right) .
$$

To get some insight for the inner maximization, we take a partial derivative of $\tilde{U}_{\tilde{\mathbf{h}}}\left(P_{T}, \alpha\right)$ over $\alpha$ and obtain,

$$
\begin{aligned}
\frac{\partial \tilde{U}_{\tilde{\mathbf{h}}}\left(P_{T}, \alpha\right)}{\partial \alpha}= & \frac{P_{T}|| \tilde{\mathbf{h}} \|^{2}}{\left(P_{T}+P_{C}\right)\left(1+\alpha P_{T}|| \tilde{\mathbf{h}} \|\left.\right|^{2}\right)} \cdot\left(1-F_{\| \tilde{\mathbf{h}}+w||}(\sqrt{\alpha} \mid \tilde{\mathbf{h}} \|)\right) \\
& -\frac{\ln \left(1+\alpha P_{T}|| \tilde{\mathbf{h}} \|^{2}\right)}{P_{T}+P_{C}} \cdot f_{\|\tilde{\mathbf{h}}+w\|}(\sqrt{\alpha}|| \tilde{\mathbf{h}} \|) .
\end{aligned}
$$

The first term in (32) is a monotonically decreasing function of $\alpha$, while the absolute value of the second term is a monotonically increasing function of $\alpha$, where $\alpha \in(0,1]$. Hence, the partial derivative is monotonically decreasing. The partial derivative has the positive supremum at $\alpha=0$, and the infimum at $\alpha=1$. If the infimum of the partial derivative is non-negative, i.e., if

$$
\frac{P_{T}\|\tilde{\mathbf{h}}\|^{2}}{\left(1+\left.P_{T}|| \tilde{\mathbf{h}}\right|^{2}\right) \ln \left(1+\left.P_{T}|| \tilde{\mathbf{h}}\right|^{2}\right)} \geq \frac{f_{\|\tilde{\mathbf{h}}+w\| \mid}(|| \tilde{\mathbf{h}} \|)}{1-F_{|| \tilde{\mathbf{h}}+w||}(\| \tilde{\mathbf{h}} \mid)},
$$

then $\tilde{U}_{\tilde{h}}\left(P_{T}, \alpha\right)$ is monotonically increasing and hence the maximum $U_{\tilde{h}}\left(P_{T}, \alpha\right)$ is achieved with $\alpha=1$. Here, the left hand side is a decreasing function of $P_{T}$, and hence the condition (33) is equivalent to $P_{T} \leq A$, where

$$
A=g^{-1}\left(\frac{f_{|| \tilde{\mathbf{h}}+w \| \mid}(\|\tilde{\mathbf{h}}\|)}{1-F_{\|\tilde{\mathbf{h}}+w\| \mid}(\| \tilde{\mathbf{h}}||)}\right),
$$


and

$$
g(x)=\frac{x\|\tilde{\mathbf{h}}\|^{2}}{\left(1+x|| \tilde{\mathbf{h}} \|^{2}\right) \ln \left(1+x|| \tilde{\mathbf{h}} \|^{2}\right)} .
$$

In this case, the joint maximization (31) becomes

$$
\tilde{U}_{\tilde{\mathbf{h}}}^{*}=\max _{P_{T}} \frac{\ln \left(1+P_{T}\|\tilde{\mathbf{h}}\|^{2}\right)}{P_{T}+P_{C}} \cdot\left(1-F_{\|\tilde{\mathbf{h}}+w\|}(\|\tilde{\mathbf{h}}\|)\right) \text {. }
$$

As $\left(1-F_{\|\tilde{\mathbf{h}}+w\|}(|| \tilde{\mathbf{h}}||)\right)$ does not depend on $P_{T}$, and hence this maximization problem over $P_{T}$ is essentially the same problem as the one in Theorem 1; $P_{T}^{*}$ and $R^{*}$ are readily obtained from Theorem 1 as

$$
P_{T}^{*}=\left(\frac{1}{U_{\mathbf{h}}^{*}}-\frac{1}{\|\tilde{\mathbf{h}}\|^{2}}\right)^{+} \quad \text { and } \quad R^{*}=\ln \left(1+P_{T}^{*}\|\tilde{\mathbf{h}}\|^{2}\right) .
$$

On the other hand, if the partial derivate is negative at $\alpha=1$, there exists an optimal $\alpha=\alpha^{*} \in(0,1)$ that maximizes $U_{\tilde{\mathrm{h}}}\left(P_{T}, \alpha\right)$, and $\alpha^{*}$ satisfies the following:

$$
\frac{P_{T}|| \tilde{\mathbf{h}} \|^{2}}{\left(1+\alpha^{*} P_{T}|| \tilde{\mathbf{h}} \|^{2}\right) \ln \left(1+\alpha^{*} P_{T}|| \tilde{\mathbf{h}} \|^{2}\right)}=\frac{f_{\|\tilde{\mathbf{h}}+w \mid\|}\left(\sqrt{\alpha^{*}}|| \tilde{\mathbf{h}} \|\right)}{1-F_{\|\tilde{\mathbf{h}}+w\|}\left(\sqrt{\alpha^{*}}|| \tilde{\mathbf{h}} \|\right)} .
$$

Here we note that $\alpha^{*}$ is a function of $P_{T}$, and hence we denote $\alpha^{*}=\alpha^{*}\left(P_{T}\right)$. Then, the joint maximization (31) now becomes

$$
\tilde{U}_{\tilde{h}}^{*}=\max _{P_{T}} \tilde{U}_{\tilde{h}}\left(P_{T}, \alpha^{*}\left(P_{T}\right)\right) .
$$

\section{Conflicting interests}

The authors declare that they have no competing interests.

${ }^{1}$ For better mathematical representations and for convenience of analysis, we use "nats" instead of "bits," for the unit of information. 1 nats $=\log _{2} e$ bits.

${ }^{2}$ This assumption can be justified as in the following. As mentioned earlier in this paper, because of the flashlight effect, the channel state used for demodulation, $\mathbf{h}$ and the one reported to the link adaptation controller, $\tilde{\mathbf{h}}$, are not necessarily the same. However, the link adaptation controller can obtain $\mathbf{h}$ from the receiver once the demodulation is finished. Therefore the link adaptation controller can determine the distribution of $\boldsymbol{w}$.

\section{Author details}

'Dallas Telecomm. R\&D Center, Samsung Telecomm. America, Dallas, Texas, 75082, USA ${ }^{2} E E C S$ Department of Electrical Engineering and Computer Science University of Kansas, Lawrence, 66045, USA

Received: 29 June 2011 Accepted: 23 November 2011

Published: 23 November 2011
References

1. 3GPP TR 36814, Evolved Universal Terrestrial Radio Access (E-UTRA); Further advancements for E-UTRA Physical layer aspects: Physical channels and modulation v1.0.0 (Feb. 2009)

2. K Lahiri, A Raghunathan, S Dey, D Panigrahi, Battery-driven system design: a new frontier in low power design. in Proc Intl Conf on VLSI Design, Bangalore, India, 261-267 (Jan. 2002)

3. GW Miao, N Himayat, Li Y, A Swami, Cross-layer optimization for energyefficient wireless communications: a survey 9(4), 529-542 (2009)

4. S Verdu, Spectral efficiency in the wideband regime. IEEE Trans Inf Theory. 48(6), 1319-1343 (2002). doi:10.1109/TIT.2002.1003824

5. F Meshkati, HV Poor, SC Schwartz, NB Mandayam, An energy-efficient approach to power control and receiver design in wireless networks. IEEE Trans Commun. 5(1), 3306-3315 (2006)

6. S Cui, AJ Goldsmith, A Bahai, Energy-constrained modulation optimization. IEEE Trans Wirel Commun. 4(5), 2349-2360 (2005)

7. G Miao, N Himayat, Li Y, D Bormann, Energy-efficient design in wireless OFDMA. in Proc IEEE Conf Commun. ICC' 2008 (2008)

8. GW Miao, N Himayat, Li GY, S Talwar, Low-complexity energy-efficient OFDMA. in Proc IEEE Conf Commun. ICC' 2009 1-5 (June 2009)

9. G Miao, N Himayat, Y Li, Energy-efficient link adaptation in frequencyselective channels. IEEE Trans Commun. 58(2), 545-554 (2010)

10. P Marsch, G Fettweis, Uplink comp under a constrained backhaul and imperfect channel knowledge. IEEE Trans Wirel Commun (2010) (Submitted)

11. L Liu, Y Nam, J Zhang, Proportional fair scheduling for multi-cell multiuser mimo systems, in Proc CISS (Princeton, NJ, May 2010)

12. L Liu, J Zhang, J-C Yu, J Lee, Intercell Interference Coordination through Limited Feedback, Int. J Digit Multimedia Broadcasting (2010). Article ID 134919, 7 pages, 2010

13. TM Cover, JA Thomas, Elements of Information Theory, (Wiely, 1991)

14. Y Nam, PK Gopala, H El Gamal, Resolving collisions via incremental redundancy: Arq diversity, in Proc INFOCOM (Anchorage, AK, May 2007)

doi:10.1186/1687-1499-2011-184

Cite this article as: Nam et al.: Link adaptation for energy-efficient uplink coordinated multi-point receptions. EURASIP Journal on Wireless Communications and Networking 2011 2011:184.

\section{Submit your manuscript to a SpringerOpen ${ }^{\circ}$ journal and benefit from:}

- Convenient online submission

- Rigorous peer review

- Immediate publication on acceptance

- Open access: articles freely available online

- High visibility within the field

- Retaining the copyright to your article

Submit your next manuscript at springeropen.com 